\section{Global Proceedings Repository \\ American Research Foundation}

ISSN 2476-017X

Available online at http://proceedings.sriweb.org

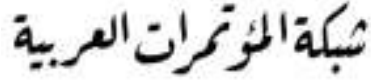

http://arab.kmshare.net/

The Ninth International Scientific Academic Conference

Under the Title "Contemporary trends in social, human, and natural sciences"

$$
\begin{aligned}
& \text { المؤتمر العلمي الاكاديمي الدولي التاسع } \\
& \text { تحت عنوان "الاتحاهات المعاصرة في العلوم الاجتماعية، الانسانية، والطبيعية" } \\
& 17 \text { - } 18 \text { يوليو - تموز } 2018 \text { - اسطنبول - تركيا }
\end{aligned}
$$

http://kmshare.net/isac2018/

\title{
Investigating the Usability of 3D Interactive Mobile Applications: The Dental Clinic Case
}

\author{
Hasan Alhajhamad ${ }^{a}$, Hadi Khalilia ${ }^{b}$, Derar Eleyan ${ }^{c}$ \\ Palestine Technical University, Tulkarim, Palestine

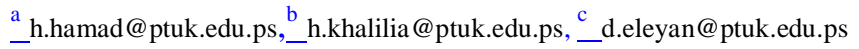

\begin{abstract}
In this paper we analyze the UX/UI of an interactive dental clinic system. It is a system which helps dentist to keep track with patients' dental problems and take care of their oral health. The visualization way of data regarding the patient dental information is essential for the treatment plan and for the future use. The main disadvantage of current applications is time consumption. One more limitation is loss of patient information. The main purpose for us to choose this dental clinic is to improve current systems so that it will become easier for the doctor to access their patients' information.
\end{abstract}

Keywords: human-computer interaction, usability, educational applications, 3-dimensional user interface, user experience. 


\section{Global Proceedings Repository \\ American Research Foundation}

ISSN 2476-017X

ARF

Available online at http://proceedings.sriweb.org

\section{Introduction}

The interactive dental clinic software is user-friendly software based on UX and UI specifications. The main objectives of the system are which shows and helps you to collect most of the information about hospitality and Services the system is very simple in design and to implement. The system requires very low system resources and the system will work in almost all configurations. Patients are easily allocated to the doctors. Doctors Search is possible, Today's patient list help doctors to search their patients(Ikram, Liaquat, Zafar, \& Baker, 2009).

Medical applications are beneficial for patients and doctors, and both have different requirements for the application they use. A person can reduce the complexity of the steps involved in the healthcare industry by using the best medical and medical applications.

The use of mobile communication devices has resulted in increased patient participation, which means that more people are focusing on improving their health and well-being, such as following a physicianrecommended diet, regular routines and respect their own appointments.

Since 2013, medical applications have attracted more attention from people in the market and their scope started increasing. Regardless of whether you belong to a health post such as doctor, patient, or any other person related to the medical industry, using an application based on the medical industry, the person will always be confronted with easy solutions to his various problems.

\section{Previous work}

There are a few existing applications that target dental care. One application is Dental Corpus Anatomy Lite (Dental Corpus, 2017), developed by Dental Corpus developers group. This mobile application is a demo version of the tooth. In this dissection, the only available tooth release is the mandible.

Dental Corpus Dental Anatomy is an interactive tool for dental students and health professionals. The purpose of this application is to help understand and visualize the complexity of anatomy and to learn detailed information about the size, morphology and development of mandibular dogs. Available tooth is a 3D model created with information on dental morphology.

Doctor on Demand (Chan, Torous, Hinton, \& Yellowlees, 2016) is another application when you are in a hurry and without the hassle of the waiting room. Communicate in minutes with certified doctors and PHD transcribers via live video.

In addition, the apps My Chart (Fletcher, Pignatelli, Jimenez-Galindo, \& Ghosh-Jerath, 2016) have facilitated which sets the results of your medical analysis, information about current traffic, current medications, the date of your vaccinations, and more in the palm of your hand on your mobile device. Using my chart, you can: Review your medical information, stay in touch with your doctor, organize upcoming visits and review the medical information of your family member.

Finally, the Application Dental (Mustaza, Lim, Ghani, \& Mariam, 2016) study is an integrated educational tool, especially aimed at students, dentists, and dentistry educators. 


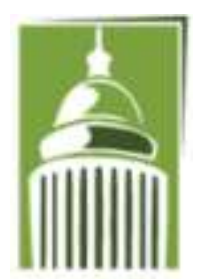

ARF

\section{Global Proceedings Repository \\ American Research Foundation}

ISSN 2476-017X

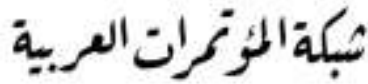

http://arab.kmshare.net/

Available online at http://proceedings.sriweb.org

The applications that are focused on the field of dentistry are few. Therefore, we should focus on more applications in the field of dental dentistry and organizing the work of dental clinics.

\section{Methodology}

This section explains of the framework of the dental clinic. Figure 1 describes the phases of the application development.

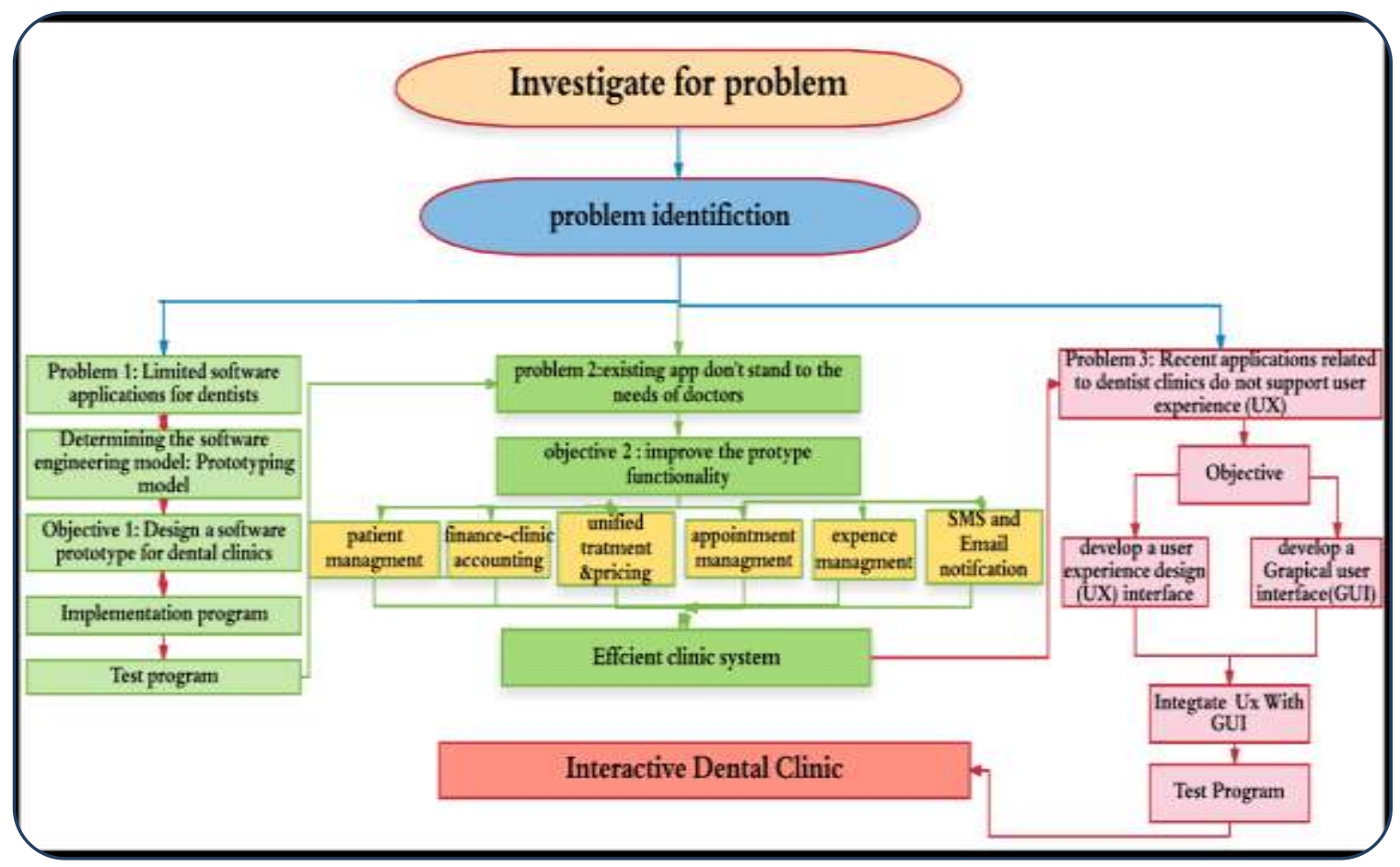

Figure 2: Interactive dental clinic app framework

\subsection{Phase1: Problem Identification}

The first step in the problem solving and decision-making process is to identify and define the problem (Gilboe \& Scott, 1991). A problem can be regarded as a difference between the actual situation and the desired situation. This means that in order to identify a problem the team must know where it is meant to be and have a clear understanding of where it currently is in relation to the perceived problem. Therefore, the main problems have been identified, namely: Limited software application for dentist, existingapp do not 


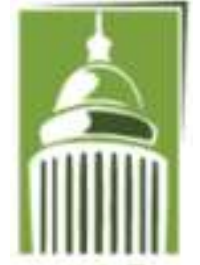

ARF

\section{Global Proceedings Repository \\ American Research Foundation}

ISSN 2476-017X

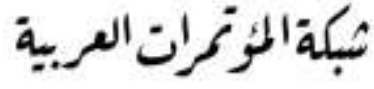

http://arab.kmshare.net/

Available online at http://proceedings.sriweb.org

stand the needs of doctors and recent application related to dentist clinic do not support user experience (UX).

Progress in information technology has changed our daily lives. The use of computers and the Internet is part of the daily activity of humanity. The use of computers is growing among dentists and doctors. Information is constantly updated on the Internet, helping to update modern knowledge and information that help motivate doctors to conduct research activities in the field of medicine and dentistry. Computers are mostly used for office procedures such as billing and research and are often less for medical education. Numerous studies have shown that the use of computerized information system in the field of dentistry and medicine can improve the quality of care, encourages the use of evidence-based therapy and helps in updating current knowledge. The internet has been used for medical education in diverse ways including teaching of organs, diagnosis of diseases and conduct of medical examinations.

Using the World Wide Web has made access to information faster, easier and in less time, and is now one of the most important sources of information for many doctors. In developed countries the use of the Internet in health care is heavily used, while in developing countries it is slowly improving for many reasons including declining social and economic growth rate and low literacy rate.

Internet applications in dentistry are limited, so dentists need training to learn how to use web search tools and techniques in order to find high-quality information resources.

\subsection{Phase 2: Design a Prototype for Dental Clinic}

The dental clinic prototype is a version of a system or part of the system that's developed quickly to check the doctor's requirements or feasibility of some design decisions (Gilboe \& Scott, 1991). So, a prototype is useful when a doctor or developer is not sure of the requirements, or of algorithms, efficiency, business rules, response time, etc. In prototyping for dental clinic, the doctor is involved throughout the development process, which increases the likelihood of doctor acceptance of the final implementation. While some prototypes are developed with the expectation that they will be discarded, it is possible in some cases to evolve from prototype for dental clinic to working system.

Figure 2 shows the dental clinic application prototype steps, the objectives, and the implementation and examination steps for dental clinic application.

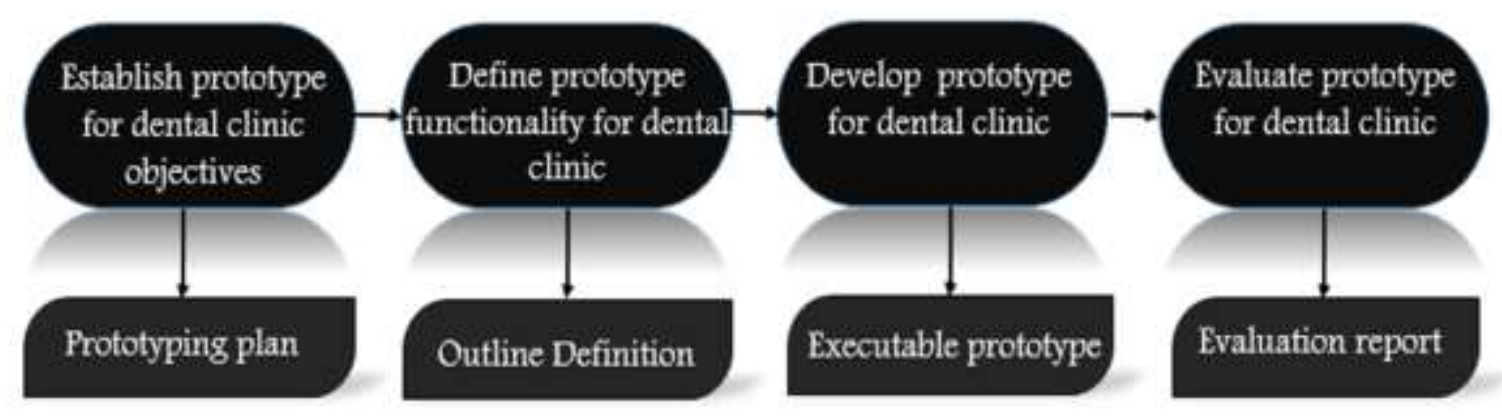

Figure 1: Prototype for Dental Clinic Development 


\section{Global Proceedings Repository \\ American Research Foundation}

ISSN 2476-017X

Available online at http://proceedings.sriweb.org

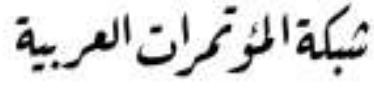

http://arab.kmshare.net/

The phases of a prototype for dental clinic are:

1- Establish prototype for dental clinic objective: The objectives of the prototype for dental clinic should be made explicit from the start of the process. It is to validate system requirements, or demonstrate feasibility, etc..

2- Define prototype functionality for dental clinic: Decide what are the inputs and the expected output from a prototype. To reduce the prototyping costs and accelerate the delivery schedule, you may ignore some functionality, such as response time and memory utilization unless they are relevant to the objective of the prototype.

3- Develop the prototype for dental clinic: The initial prototype for dental clinic is developed that includes only user interfaces.

4- Evaluate the prototype for dental clinic: Once the users are trained to use the prototype for dental clinic, they then discover requirements errors. Using the feedback both the specifications and the prototype for dental clinician be improved. If changes are introduced, then a repeat of steps 3 and 4 may be needed.

The outcome of this phase is to create simple mockups, user interfaces, flows and more. Quickly drag and drop UI elements from built-in or custom pattern libraries to create your prototype. Then, style it with fills, gradients, line styles, and text formatting. Also, create simple click-through wireframes or highly functional, rich prototypes with conditional logic, dynamic content, animations and data-driven interactions without writing a single line of code.

The functionalities this phase should perform are:

1- The login application we create is going to be multi-paged. These are some of the activities you will need: Login Page (Main Activity), signup page, logged-in page

2- After logging in or registering, you enter the main page and contain the search process and icons to navigate to other pages of the application. The create is going to be multi-paged. These are some of the activities you will need: Main Page (Main Activity), search patient page, edit profile page.

There are many ways to navigate between pages in the application. There are links in the archive, links to categories, and links to pages, internal sharing links, individual share links, admin comments links, bookmark links, and many other types of navigation links. When it comes to the serial transfer of characters, it provides many useful templates designed to connect the shackles dynamically together.

3- When the patient is searched for, the first letter of his name is written, and the list of patients' names appears from the letter that was searched on another page. The search page create is going to be multipaged. These are some of the activities you will need: Search Page (Main Activity), search names page.

4- The doctor will add information about the patient. These are some of the activities you will need: Add description page (Main Activity).

5- The add patient page create is going to be one paged. These are of the activities you will need: Add patient Page (Main Activity).

\subsection{Phase 3: Improve the prototype functionality}

The existing applications do not stand to the needs of the doctors in terms of collecting their medical history, communication with patients, and managing the data and financial expenditures etc. The interactive 


\section{Global Proceedings Repository \\ American Research Foundation}

ISSN 2476-017X

Available online at http://proceedings.sriweb.org

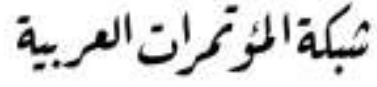

http://arab.kmshare.net/

dental clinic offers intelligent dashboard with extensive activity reports, total revenue, outstanding, patient appointments, patient visits, treatments and much more. The functionalities this phase should perform are:

1- Patient Management: Complete patient information entry like demographic, contact, family etc, view case history, lab tests, patient visits, payment details and track patients dental health progress. Patient management would include: Adding patients and family members, phone number, case number, blood group, email id etc. Adding medication for patient and his/her diseases, last update date. A record of a patient's oral health, general health, medical conditions, and medical care, including surgeries and medication use.

2- Reports management: An essential feature of interactive dental clinic, its enables users to view reports patient-wise, payment-wise, treatment-wise.it can give you list of patient, payment, and treatment handled in different financial years. Simple interface and few fields make it an easier task to get reports as per your requirement.

3- Finance - Clinic accounting: Streamline electronic claim submission, manage credit and debit card payments, create and send billing statements on demand, collect more payments in less time-with less difficulty, capture treatment charges, create and print bills and receipts, see which patient owes what and for how long.

4- Unified Treatments and Pricing: Central administrative controls to change rates for consultations, treatments, procedures and more, new treatments can be added along with costs and kept active or deactivated in line with ongoing offers.

5- Appointment management at its best: Patients accept treatment more readily and leave more satisfied when their plan is well organized, and they have fewer financial questions.

a. View Appointment: Dashboard for the doctors' slot on a single screen, Quick appointment system with the appointment scheduler, Reminder for follow-up call for confirmed dental appointment

b. Mobile Appointment: Option for patient to book dentist appointment online through mobile website, option for patient to book appointment through mobile app.

6- Expenses management: This is a simple tracking and budgeting software module which comes as an extra feature with interactive dental clinic. In this module, dentist's categories like phone bill, clinic space rent, electricity bill, stationary etc, they can add the respective amount in it. This will give an idea of the monthly /yearly expenses and can help in managing the finances.

7- SMS and Email notification:

a. Email/ SMS to the patient directly through interactive dental application

b. Email/SMS bill, receipt, prescription, reminder through email

c. Make patient email group and send emails to all patients in a single click in this practice management.

Dentists are busy treating patient and they hardly get any time to record the details of the patient and do the clerical and repetitive jobs. Sometimes they have a staff and their disposal but confidential information like estimation, prescription and billing is mostly done by the dentist themselves .so the challenge was to provide them with a great functional solution which will have simple and easy user interactive and the same time would be quick and less time consuming.

Interactive dental clinic management application software covers all aspects of dental office management. It can be installed on a single computer or multiple computers in a network. 


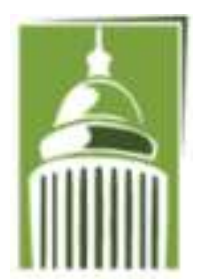

ARF

\section{Global Proceedings Repository \\ American Research Foundation}

ISSN 2476-017X

Available online at http://proceedings.sriweb.org

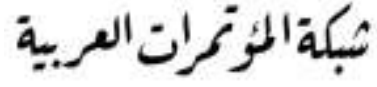

http://arab.kmshare.net/

The purpose of this software is to save time and efforts of the dentists. It will reduce the need for manual data entry by automating all of the management process.

\subsection{Phase 4: Develop a user experience design (UX) interface}

The User Experience (UX) focuses on a deep understanding of what users need, their value, capabilities and limitations. It also considers the objectives and objectives of the group managing the project. Best Practices to improve the quality of the user's interaction with the perceptions of your products and related services. GUI is a type of user interface that allows users to interact with electronic devices through graphic icons. The term came into being because the first interactive user interfaces for computers were not graphical: they were composed of text and a directed keyboard and were usually composed of commands. GUI operating systems are much easier to learn and use because the commands do not need to be backed up. In addition, users do not need to know the programming languages. Because of their ease of use, operating systems have become the dominant operating systems graphical user interface used by today's end users, they are used in many portable devices such as readers MP3, game consoles, offices and industrial controls.

The treatment user interface can be viewed as a guideline for a dental treatment or examination, a chart for patients and dentists. It is also a training tool for dental professionals, orthodontists and students to strengthen their experience.

In this application has been used by a set of icons and 3D object to facilitate the doctor to carry out operations, for example, the search icon to facilitate the doctor to search for the patient if he was previously.

The user experience considers the expectations and desires of the user to achieve his goal without much effort in a simple, easy and fluid environment.

In this application will be used UX to facilitate the doctor access to information and deal with it easily by clicking on the icons, for example, when pressing on the tooth, it will review all possible operations from the tooth extraction and other things. UX and GUI make applications easy to use. At the same time, users can easy to understand. Although the UI and UX are two different things, they are essentially two sides of the same coin design application. Indeed, while working on the user interface, you can also take care of UX problems and in reverse.

To log in to the admin, retrieving lost passwords. Each of these activities is managed on the same page, usually called the log in page. After logging in, you can access your main page that contain icons to access other pages of the application. When the doctor wants to add new patient, he will click the icon add patient and fill information about him. When the doctor wants to search for a patient , he writes the first letter of his name then press icon search, it shows the list of patients' names appears from the letter. When the doctor want adds an appointment for patient, he will click the icon add an appointment and fill information. When the doctor wants to edit information about patient, he will click the icon edit patient and edit information about him. When the doctor wants to know all information about patient, he will click the icon show details then show information about him. 


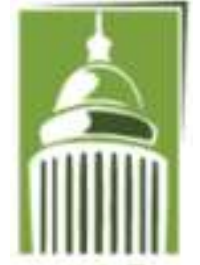

ARF

\section{Global Proceedings Repository \\ American Research Foundation}

ISSN 2476-017X

Available online at http://proceedings.sriweb.org

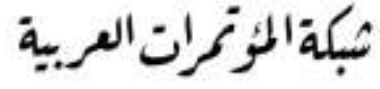

http://arab.kmshare.net/

\subsection{Phase 5: App Testing}

Testing is one of the most important aspects of application development. In this application designers release the application in the community test application before releasing it to the public. This is done to obtain important comments from real, independent and objective users of the application. It is common for designers to modify the app based on the testers' comments. By testing the application, developers are trying to avoid the future where it becomes necessary to start developing the application from zero.

Manual testing is a test process that is performed manually to detect defects without the use of automated tools or scripts. A test plan document as a guide for the test process is prepared to obtain complete test coverage.

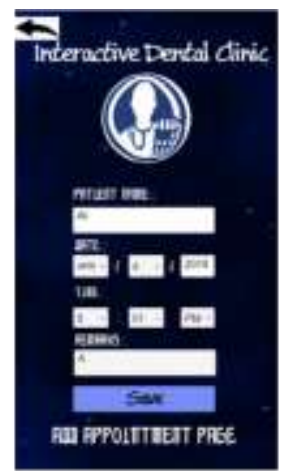

Add appointment

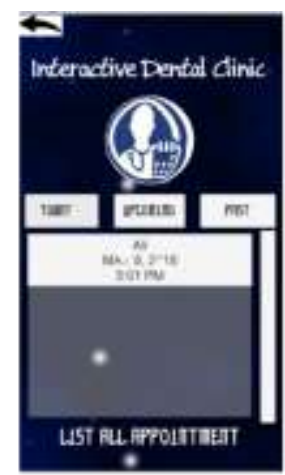

List all appointment
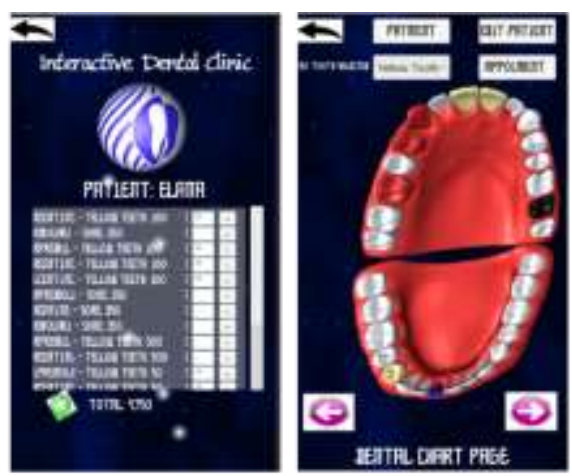

Payment description Dental chart

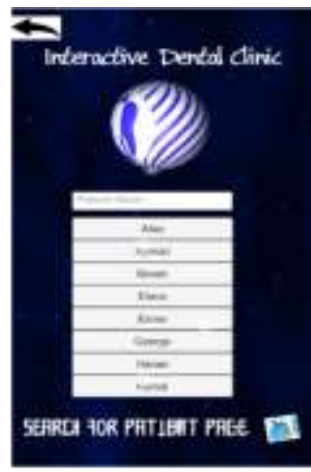

Search for patient

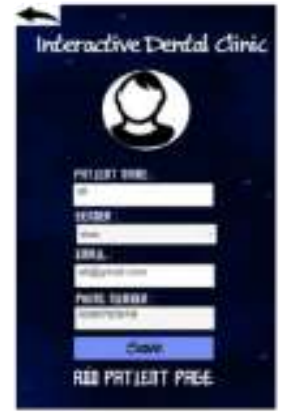

Add patient

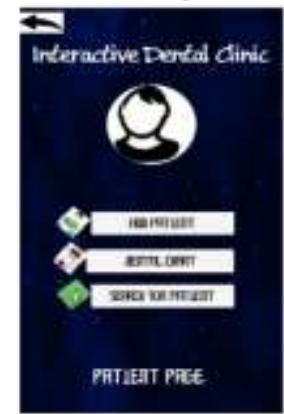

Patient page

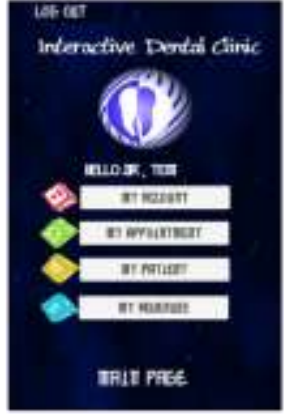

Main page

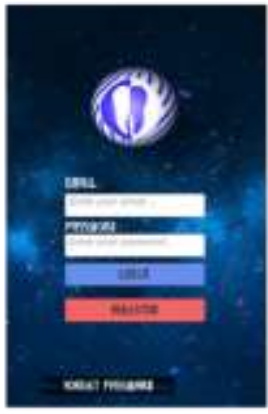

Log-in page

Figure 2: Implementation for Interactive Dental Clinic app pages

\section{Discussion}

User Interface Design is concerned with how users add information to the system and with how the system presents information back to them. Data Design is concerned with how the data is represented and stored within the system. Finally, Process Design is concerned with how data moves through the system, 


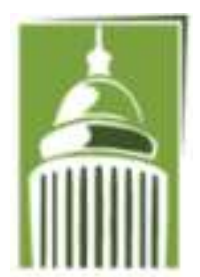

ARF

\section{Global Proceedings Repository \\ American Research Foundation}

ISSN 2476-017X

Available online at http://proceedings.sriweb.org

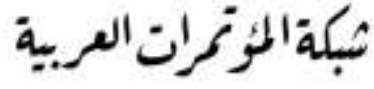

http://arab.kmshare.net/

and with how and where it is validated, secured and/or transformed as it flows into, through and out of the system. At the end of the system design phase, documentation describing the three sub-tasks is produced and made available for use in the next phase.

In this section, the next step is systems design, system design is the process of defining the architecture, modules, interfaces, and data for a system to satisfy specified requirements. Systems design could be seen as the application of systems theory to product development. There is some overlap with the disciplines of systems analysis, systems architecture and systems engineering. The system requirements such as hardware and software, the implementation and coding the system components describe the development tools that have been used in this system. Table 1 describes the hardware and software requirements of the Interactive Dental Clinic.

Table 1: Hardware and software requirements

\begin{tabular}{|l|l|}
\hline Device & Description \\
\hline Processor & Intel(R) Core(TM) i74005U CPU @ 1.70 GHz \\
\hline RAM & $8 \mathrm{~GB}$ \\
\hline System Type & 64-bit Operating System. \\
\hline Operating System & Windows 7 Ultimate. \\
\hline Software & \\
\hline Microsoft Visual Studio & Version 13.030 \\
\hline Unity & Version $2017.2 .0 \mathrm{f3}$ \\
\hline Blender & Version 2.79 \\
\hline
\end{tabular}

Database system in this app included XML files. An XML database is a database that stores data in XML format. This type of database is suited for businesses with data in XML format and for situations where XML storage is a practical way to archive data, metadata and other digital resources.

Figure 3 describes the app's screens implementation. All these screens are implemented in a way that When the Interactive Dental Clinic app starts, a splash screen shows up. After that, the user registers in to the application. After $\log$ in, we go to the main page that have four buttons. In my account, doctor can edit his information and change password. When we click to the patient button, we get three options like add patient, dental chart and search for patient. If we click to the search for patient button, we get the all patient that register. After that, we select the name of patient and get his dental chart. We have the payment for every teeth and doctor can make discount. Add appointment button, doctor can add and list all appointment.

\section{Conclusion}

In this paper we develop a mobile application using 3D object to facilitate the doctor dealing with information from save, modify and delete. Using of 3D interactive dental clinic app, where the software 


\section{Global Proceedings Repository \\ American Research Foundation}

ISSN 2476-017X

Available online at http://proceedings.sriweb.org

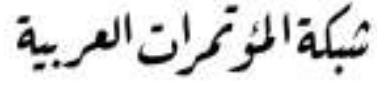

http://arab.kmshare.net/ ARF

support user experience and user interface, containing several pages each page has a certain function such that, when logging-in and go to main page then select patient page this contains many functions. The work in this project provides future applications in several areas, these include: Study new ways to build system models for interactive dental clinic, develop the application to link to cloud system, develop the application to cover the patient side, add multi-language support. Add the feature of sending SMS to patients to alert them for appointment.

\section{References}

Chan, S., Torous, J. B., Hinton, L., \& Yellowlees, P. M. (2016). Psychiatric Apps: Patient Self-Assessment, Communication, and Potential Treatment Interventions. In e-Mental Health (pp. 217-229). Cham: Springer International Publishing. https://doi.org/10.1007/978-3-319-20852-7_11

Dental Corpus. (2017). Dental Corpus Anatomy Lite. Retrieved from http://www.dentalcorpus.com/

Fletcher, R., Pignatelli, N., Jimenez-Galindo, A., \& Ghosh-Jerath, S. (2016). Development of smart phone tools for printed diagnostics: Challenges and solutions. In 2016 IEEE Global Humanitarian Technology Conference (GHTC) (pp. 701-708). IEEE. https://doi.org/10.1109/GHTC.2016.7857355

Gilboe, D. B., \& Scott, D. A. (1991). Computer systems for dental practice management. A new generation of independent dental software. Journal (Canadian Dental Association), 57(10), 782-786. Retrieved from http://www.ncbi.nlm.nih.gov/pubmed/1742661

Ikram, A., Liaquat, S., Zafar, M., \& Baker, N. (2009). Experiences in Design and Development of ContextAware IMS-Based Multimedia Services for Ubiquitous Environments. Next Generation Mobile Applications, Services and Technologies, 2009. NGMAST '09. Third International Conference On. https://doi.org/10.1109/NGMAST.2009.67

Mustaza, T. A., Lim, T. W., Ghani, A., \& Mariam, S. (2016). Mobile applications at the dentist. International Journal on E-Learning and Higher Education, 4, 16-28. 